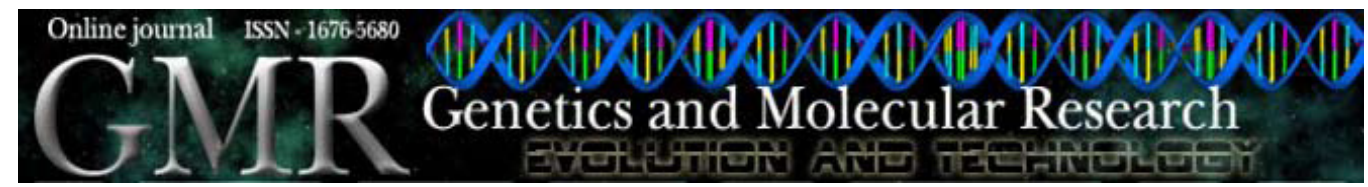

\title{
Differential expression of E-cadherin gene in human neuroepithelial tumors
}

\author{
F.J.N. Motta ${ }^{1,2}$, E.T. Valera $^{3}$, A.K.B. Lucio-Eterovic ${ }^{3}$, R.G.P. Queiroz ${ }^{3}$, \\ L. Neder ${ }^{4}$, C.A. Scrideli ${ }^{3}$, H.R. Machado ${ }^{5}$, C.G. Carlotti-Junior ${ }^{5}$, \\ S.K.N. Marie $^{6}$ and L.G. Tone ${ }^{3}$ \\ ${ }^{1}$ Departamento de Genética, Faculdade de Medicina de Ribeirão Preto, \\ Universidade de São Paulo, Ribeirão Preto, SP, Brasil \\ ${ }^{2}$ Universidade Federal do Piauí, Campus Ministro Reis Veloso, Parnaíba, PI, Brasil \\ ${ }^{3}$ Departamento de Puericultura e Pediatria \\ Faculdade de Medicina de Ribeirão Preto, Universidade de São Paulo, \\ Ribeirão Preto, SP, Brasil \\ ${ }^{4}$ Departamento de Patologia, Faculdade de Medicina de Ribeirão Preto, \\ Universidade de São Paulo, Ribeirão Preto, SP, Brasil \\ ${ }^{5}$ Departamento de Anatomia e Cirurgia, \\ Faculdade de Medicina de Ribeirão Preto, Universidade de São Paulo, \\ Ribeirão Preto, SP, Brasil \\ ${ }^{6}$ Departamento de Neurologia, Faculdade de Medicina, \\ Universidade de São Paulo, São Paulo, SP, Brasil \\ Corresponding author: F.J.N. Motta \\ E-mail:motta@ufpi.edu.br
}

Genet. Mol. Res. 7 (2): 295-304 (2008)

Received December 18, 2007

Accepted February 8, 2008

Published April 8, 2008

\begin{abstract}
Cadherins are cell-to-cell adhesion molecules that play an important role in the establishment of adherent-type junctions by mediating calcium-dependent cellular interactions. The $C D H 1$ gene encodes the transmembrane glycoprotein E-cadherin which is important in maintaining homophilic cell-cell adhesion in epithelial tissues. E-cadherin interacts with catenin proteins to maintain tissue architecture. Structural defects or loss of expression of E-cadherin have been reported as a common feature in several human cancer types. This study aimed to evaluate the expression of E-cadherin and their correlation with clinical features in microdissected brain
\end{abstract}


tumor samples from 81 patients, divided into 62 astrocytic tumors grades I to IV and 19 medulloblastomas, and from 5 white matter non-neoplasic brain tissue samples. E-cadherin $(\mathrm{CDH1})$ gene expression was analyzed by quantitative real-time polymerase chain reaction. Mann-Whitney, Kruskal-Wallis, Kaplan-Meir, and logrank tests were performed for statistical analyses. We observed a decrease in expression among pathological grades of neuroepithelial tumors. Non-neoplasic brain tissue showed a higher expression level of $C D H 1$ gene than did neuroepithelial tumors. Expression of Ecadherin gene was higher in astrocytic than embryonal tumors $(\mathrm{P}=$ 0.0168). Low-grade malignancy astrocytomas (grades I-II) showed higher $\mathrm{CDH} 1$ expression than did high-grade malignancy astrocytomas (grades III-IV) and medulloblastomas $(\mathrm{P}<0.0001)$. Non-neoplasic brain tissue showed a higher expression level of $C D H 1$ gene than grade I malignancy astrocytomas, considered as benign tumors $(\mathrm{P}=0.0473)$. These results suggest that a decrease in E-cadherin gene expression level in high-grade neuroepithelial tumors may be a hallmark of malignancy in dedifferentiated tumors and that it may be possibly correlated with their progression and dissemination.

Key words: Cancer; Neuroepithelial tumors; $C D H 1$ expression; Real-time polymerase chain reaction

\section{INTRODUCTION}

Neuroepithelial tumors are central nervous system (CNS) neoplasms that include a series of primary brain tumors that embody astrocytic, ependymal, choroid plexus, pineal parenchymal, and embryonal tumors (Louis et al., 2007). CNS neoplasms are the most frequent solid tumors of infancy and represent the second most common in children, only exceeded by the leukemias, and constitute the third cause of death in adulthood (Pötter et al., 1998; Behin et al., 2003).

Tumors of neuroepithelial origin represent a heterogeneous group of intracranial neoplasms with distinct features that control their ontogeny, pattern of dissemination and invasion, age of occurrence, clinical outcome, and prognosis. These features may reflect the complexity of the molecular and genetic alterations in pathways involved in the onset, maintenance and progression of CNS tumors (Strother et al., 2002). The pathways commonly cited in the literature as involved in the development of CNS tumors include: cell cycle control, angiogenesis (Khatua et al., 2003), and apoptosis (Pingoud-Meir et al., 2003), cell migration and adhesion molecules (Munaut et al., 2003).

Cadherins are cell-to-cell adhesion molecules that play an important role in the establishment of adherent-type junctions by mediating calcium-dependent cellular interactions, characterized by extracellular cadherin repeats of approximately 110 amino acid residues, discovered by Takeichi in 1991, which play an important role in histogenesis and morphogenesis (Pokutta and Weis, 2007). The differential expression of cadherins is particularly complex in the context of the CNS (Redies, 2000; Shapiro et al., 2007). 
The $\mathrm{CDH} 1$ gene encodes the transmembrane glycoprotein E-cadherin which is important in maintaining homophilic cell-cell adhesion in epithelial tissues. Alterations in Ecadherin expression have been related to several cancer types and correlated with pathological features such as poor tumor differentiation, infiltrative growth, lymph node metastasis, and decreased patient survival (Hirohashi, 1998; Hazan et al., 2004). The cytoplasmic catenins form a complex with E-cadherin by binding to the carboxy-terminal domain of this molecule and making a link to actin cytoskeleton that helps to maintain cell adhesion (Yagi and Takeichi, 2000; Wijnhoven et al., 2000). The gene $C D H 1$, localized on chromosome 16q22.1, was reported as an invasion-suppressor gene, though in in vitro assays (Van Aken et al., 2001).

Alterations in E-cadherin-mediated cell-cell adhesion are associated with the phenotype known as epithelial-mesenchymal transition. The essential features of epithelial-mesenchymal transition are the loss of intercellular contacts and the enhancement of cell motility, thereby leading to the release of cells from the parent epithelial tissue. The resulting mesenchymal-like phenotype is suitable for tumor invasion and dissemination, allowing metastatic progression to proceed (Becker et al., 2007; Guarino et al., 2007). Decrease or loss of expression of E-cadherin is often reported as an early event in the development of many tumors, such as gastric cancer (Graziano et al., 2003; Yi Kim et al., 2007), breast carcinoma cell lines (Lombaerts et al., 2006) and prostate cancer (Mol et al., 2007).

Although E-cadherin is a well-known repressor of invasion, little is known about the expression of this cell-cell adhesion molecule in brain tumors and their correlations with clinical and pathologic features of neuroepithelial tumors. Fewer immunohistochemical studies have reported a decrease or loss of expression of E-cadherin in brain tumors, especially in high-grade tumors (Schwechheimer et al., 1998; Utsuki et al., 2002, 2004). The current study investigates the pattern of mRNA expression levels of $C D H 1$ in a series of primary neuroepithelial tumors of children and adults by quantitative real-time polymerase chain reaction (PCR) and their association with survival in neuroepithelial tumors.

\section{MATERIAL AND METHODS}

\section{Patients analyzed}

For this study, 81 fresh-frozen microdissected tumor samples were obtained from gross total surgical resection including 62 astrocytic tumors, grades I-IV, and 19 medulloblastomas, tumors of embryonic cell linage, according to the WHO classification (Louis et al., 2007), from subjects admitted for diagnosis and treatment in Hospital das Clínicas da Faculdade de Medicina de Ribeirão Preto-USP and from Hospital das Clínicas da Faculdade de Medicina de São PauloUSP, São Paulo, Brazil. Five samples of microdissected non-neoplasic brain tissues (white matter) were obtained from patients who had undergone surgery for medical treatment of epilepsy. This genetic study was approved by the Research Ethics Committee of the University Hospital, Faculty of Medicine of Ribeirão Preto, University of São Paulo (process number 11458/2006). All samples obtained were submitted to microdissection; this procedure was performed to discard necrotic tissues and thus assure the quality of our samples. Patients' mean age was 10.8 years for grade I astrocytomas, 32.3 years for grade II astrocytomas, 30.2 years for grade III astrocytomas, 52.1 years for grade IV astrocytomas (also known as glioblastomas), and 8.9 years for medulloblastomas. Table 1 presents the histological diagnosis and the number of children and adults enrolled in this study. 


\begin{tabular}{|c|c|c|c|c|}
\hline \multirow[t]{2}{*}{ Diagnosis } & \multicolumn{2}{|c|}{ Pediatric cases } & \multicolumn{2}{|c|}{ Adult cases } \\
\hline & $\mathrm{N}$ & $\%$ & $\mathrm{~N}$ & $\%$ \\
\hline Astrocytoma grade I & 18 & $45 \%$ & 3 & $7.3 \%$ \\
\hline Astrocytoma grade II & 0 & $0 \%$ & 10 & $24.4 \%$ \\
\hline Astrocytoma grade III & 3 & $7.5 \%$ & 7 & $17.1 \%$ \\
\hline $\begin{array}{l}\text { Astrocytoma grade IV } \\
\text { (glioblastoma) }\end{array}$ & 1 & $2.5 \%$ & 20 & $48.8 \%$ \\
\hline Medulloblastoma & 18 & $45 \%$ & 1 & $2.4 \%$ \\
\hline
\end{tabular}

\section{RNA extraction and cDNA synthesis}

Total RNA was extracted with TRIZOL Reagent (Invitrogen, Carlsbad, CA, USA) and cDNA was constructed using the High Capacity Kit (Applied Biosystems, Foster City, CA, USA), according to manufacturer protocol.

\section{Quantitative real-time polymerase chain reaction}

The mRNA expression of $C D H 1$ and endogenous control gene $\beta$-glucuronidase $(G U S \beta)$ was quantified by real-time PCR with Gene Amp ${ }^{\circledR} 7500$ Sequence Detection System (Applied Biosystems). Amplification of the PCR products was obtained using on demand TaqMan $^{\circledR}$ probes (Applied Biosystems).

Blank and standard controls were run in parallel to verify the amplification within each experiment. For relative quantification of $\mathrm{CDH1}$ gene expression level, standard curves were built for each gene by considering at least three points in triplicate of a 10-fold dilution series of cDNA in water, starting from 1:10 of a volume of undiluted cDNA. The slopes observed were -3.34 for $C D H 1$ gene and -3.39 for GUS $\beta$. The coefficient of amplification was 1.99 and 1.97, respectively.

The normalized expression level of $C D H 1$ gene was determined by dividing by the expression level of GUS $\beta$ gene in the same sample. The glioblastoma cell line U343 was used as a standard control. The normalized expression level of each sample was referred to the U343 normalized value for the same gene, which was arbitrarily assumed to be 1, as reported earlier by Scrideli et al. (2003). All PCR were performed in duplicate.

\section{Statistical analysis}

We performed statistical analysis by Mann-Whitney and Kruskal-Wallis tests for comparison of gene expression among tumors of the CNS, and the Dunn multiple comparison post-test was performed to compare the differences between each neuroepithelial tumor group. Astrocytic tumors were divided into low-grade malignancy astrocytomas (grades I-II) and high-grade malignancy astrocytomas (grades III-IV). Patients with values above the median were considered as showing overexpression of the genes studied. Survival curves were performed by Kaplan-Meier and log-rank tests to evaluate the overall survival. For statistical tests, we used the GraphPad Prism Software, version 4.0 (GraphPad Software, San Diego, CA, USA). The level of significance was considered to be $\mathrm{P}<0.05$. 


\section{RESULTS}

A total of 81 patients with neuroepithelial tumors and five samples of microdissected non-neoplasic white matter were included in this study. Forty-eight subjects were male and thirty-three were female. The M:F ratio observed was 1.45 .

The pattern of expression was firstly compared between the neuroepithelial tumors according to their cell linage, astrocytic tumors (grades I-IV) versus embryonal tumors (medulloblastomas). Table 2 presents the median values and percentiles for this comparison. We observed that astrocytic tumors expressed higher levels of $C D H 1$ gene $(\mathrm{P}=0.0168$; Figure 1).

Table 2. Median and mean values of $C D H 1$ expression gene in astrocytic cell tumors and embryonic cell tumors.

\begin{tabular}{|c|c|c|c|c|}
\hline & & $\begin{array}{l}\text { Astrocytic cell tumors } \\
\qquad(\mathrm{N}=62)\end{array}$ & $\begin{array}{l}\text { Embryonic cell tumors } \\
\qquad(\mathrm{N}=19)\end{array}$ & $\mathrm{P}$ \\
\hline $\mathrm{CDHI}$ & $\begin{array}{l}\text { Median } \\
\text { P 25-75 }\end{array}$ & $\begin{array}{c}0.0260 \\
0.0060-0.0670\end{array}$ & $\begin{array}{c}0.0080 \\
0.0025-0.0285\end{array}$ & 0.0168 \\
\hline
\end{tabular}

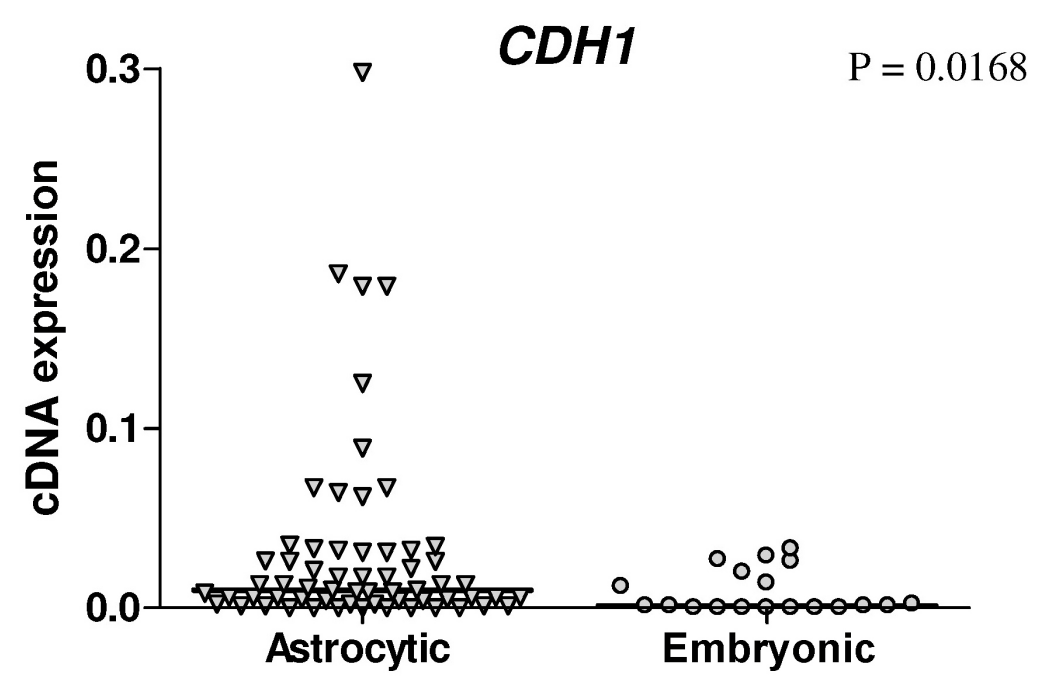

Figure 1. Differences in $C D H 1$ gene expression level among neuroepithelial tumors according to their cellular origin. The line corresponds to the median value.

To compare $\mathrm{CDH} 1$ expression level in terms of diagnosis, low-grade malignancy astrocytomas (grades I and II) and high-grade malignancy astrocytomas (grades III and IV) were evaluated as distinct groups versus non-neoplasic white matter (Table 3). Non-neoplasic brain tissue showed a higher median of expression of $\mathrm{CDH1}$ gene. Differences in $\mathrm{CDH} 1$ expression level among non-neoplasic brain tissue, high-grade malignancy astrocytomas and low-grade malignancy astrocytomas were observed $(\mathrm{P}<0.0001)$ (Figure 2A). 
Table 3. Median and mean values of $C D H 1$ expression gene in astrocytic tumors and non-neoplasic brain tissue.

\begin{tabular}{ccccc}
\hline & $\begin{array}{c}\text { Non-neoplasic brain tissue } \\
(\mathrm{N}=5)\end{array}$ & $\begin{array}{c}\text { Low-grade astrocytomas } \\
(\mathrm{N}=31)\end{array}$ & $\begin{array}{c}\text { High-grade astrocytomas } \\
(\mathrm{N}=31)\end{array}$ \\
\hline $\mathrm{CDH1}$ & Median & 0.0670 & 0.0260 & 0.0080 \\
& $\mathrm{P} 25-75$ & - & $0.0060-0.0670$ & $0.0025-0.0285$ \\
$\mathrm{P}$ & $>0.05$ & $<0.001$ & $<0.001$ \\
\hline
\end{tabular}

When we analyzed the $\mathrm{CDH} 1$ expression level only in low-grade astrocytomas and high-grade astrocytomas, this difference was maintained $(\mathrm{P}=0.0002)$ (data not shown).

Nevertheless, comparing only non-neoplasic brain tissue to grade I malignancy astrocytomas, known as a benign tumor, we observed that non-neoplasic brain tissue showed a higher expression level than did grade I astrocytomas $(\mathrm{P}=0.0473$; Figure $2 \mathrm{~B})$.

A $\quad$ CDH1 $\quad \mathrm{P}=0.0001$
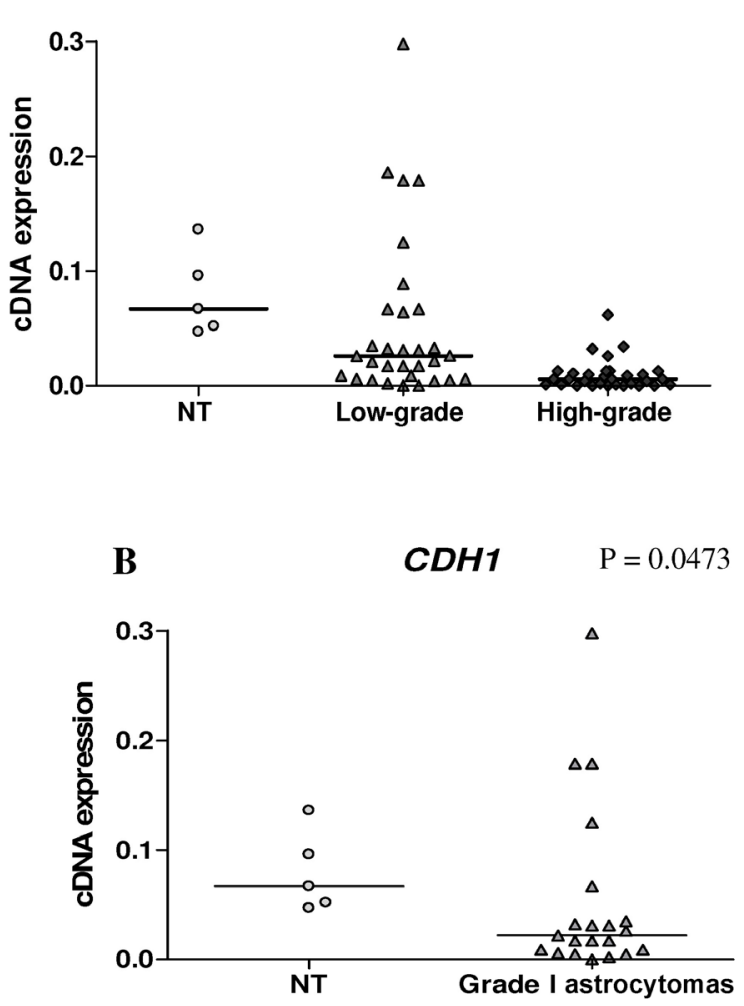

Figure 2. A. Differences in the expression levels of $C D H 1$ among non-neoplasic brain tissue (NT) and astrocytic tumors. There was a significant difference between the $C D H 1$ levels in non-neoplasic white matter and high-grade astrocytomas $(\mathrm{P}<0.0001)$. Low-grade astrocytomas also show higher $C D H 1$ expression level than high-grade astrocytomas $(\mathrm{P}<0.0001)$. B. Non-neoplasic white matter shows higher expression $C D H 1$ level than grade I astrocytomas, which show benign behavior $(\mathrm{P}=0.0473)$. The line corresponds to the median value. 
We also evaluated the expression level of $\mathrm{CDH} 1$ in the more potentially invasive groups of neuroepithelial tumors, glioblastomas and medulloblastomas, and found no differences in the level expression of E-cadherin gene $(\mathrm{P}=0.4174)$. To evaluate the impact of expression of $\mathrm{CDH}$ l gene on overall survival, the patients were divided into two groups: patients who had values above the median of expression, representing overexpression of the gene studied and patients who had a value below the median representing low expression of $\mathrm{CDH} 1$ gene. Patients with low-grade astrocytomas (grades I and II) were not included in this survival analysis because for most of them the surgical procedure was curative. No significant differences in overall survival were apparent for patients with high-grade astrocytomas and medulloblastomas with regard to $\mathrm{CDH1}$ expression (Figure 3A,B, respectively).

$\mathbf{A}$

High-grade astrocytomas - $C D H 1$
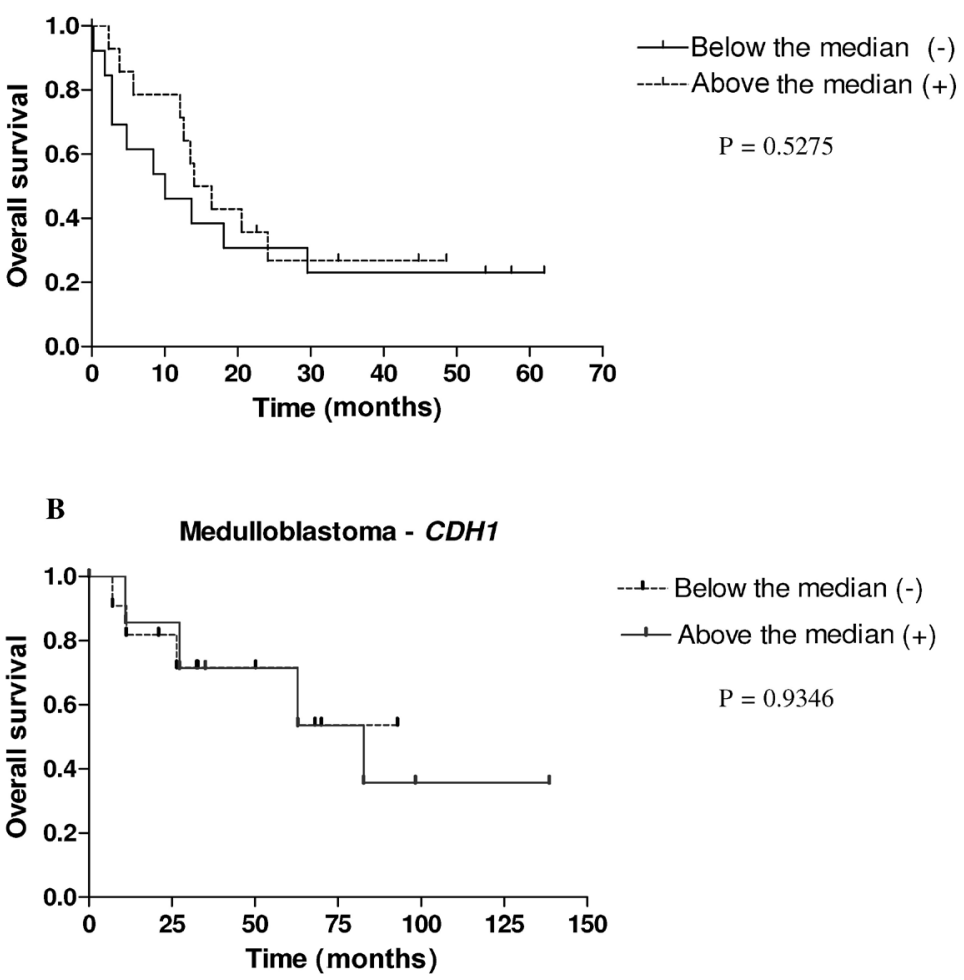

Figure 3. A. Overall survival of subjects with high-grade astrocytomas (grades III-IV) according to the decrease in expression of $\mathrm{CDH} 1$ gene. B. Overall survival of the patients with medulloblastomas according to the decrease in expression of the gene under study.

\section{DISCUSSION}

Brain tumors mostly result in death because of their aggressive and invasive properties. For cells to detach from a primary tumor site and migrate to form metastases, significant 
molecular changes in its adhesive pathway may occur (Cavallaro and Christofori, 2001). Expression of cadherin family genes has an important role in the development and progression of many cancers, including brain tumors (Howng et al., 2002).

Reduced expression of E-cadherin is regarded as one of the main molecular events involved in the dysfunction of the cell-cell adhesion system, triggering cancer invasion and metastasis (Perego et al., 2002). Our results showed that $C D H 1$ gene was less expressed in highgrade neuroepithelial tumors (glioblastomas and medulloblastomas), whereas non-neoplasic brain tissue and low-grade astrocytomas, tumors with a lower tendency for malignant spread, showed a higher $\mathrm{CDH} 1 \mathrm{expression} \mathrm{level.} \mathrm{These} \mathrm{results} \mathrm{indicate} \mathrm{that} \mathrm{E-cadherin} \mathrm{is} \mathrm{expressed} \mathrm{at}$ different levels among the malignancy grades of brain tumors.

Low $C D H 1$ expression level observed among glioblastomas and medulloblastomas, the most aggressive and dedifferentiated tumors in our series of brain neoplasias, suggests that a decrease in expression of E-cadherin gene is a hallmark of malignancy. These data correlate very well with observations in other human cancers, such as gastric cancer (Graziano et al., 2003) and breast cancer (Lombaerts et al., 2006), where a decrease or loss of E-cadherin expression has been correlated with tumor dedifferentiation and poor prognosis.

Reports about the expression of gene $\mathrm{CDH} 1$ in neuroepithelial tumors are quite rare. Some authors, using immunohistochemistry in the study of high-grade astrocytomas and medulloblastomas (Utsuki et al., 2002, 2004, respectively), demonstrated a loss of E-cadherin in these tumors displaying the invasive features of these malignant tumors. Similar results were reported previously by Asano et al. (2000) in high-grade astrocytomas (grades III-IV). In contrast, benign neuroepithelial tumors, such as chroroid plexus papilloma and benign meningiomas, showed an intense immunoreactivity for E-cadherin (Schwechheimer et al., 1998), reinforcing that the loss of expression of E-cadherin is a malignant feature.

The mechanisms involved in the downregulation of E-cadherin expression during tumor development can be accomplished by aberrant $\mathrm{CpG}$ methylation, transcriptional repressors (Garinis et al., 2002), mutations and posttranscriptional alterations (Masterson and O’Dea, 2007).

Global aberrant $\mathrm{CpG}$ methylation in $\mathrm{CDH} 1$ gene promoter was reported as a frequent epigenetic event in high-grade malignancy astrocytomas (Uhlmann et al., 2003; Yu et al., 2004), suggesting that this epigenetic mechanism may be the most important cause of loss of E-cadherin expression in astrocytic tumors. This epigenetic event is rare in medulloblastomas as shown by Frühwald et al. (2001). Interestingly, the $C D H 1$ gene is located at 16q22.1, a locus commonly deleted in medulloblastomas (Russo et al., 1999), the tumors that showed the lowest $C D H 1$ expression level in our study.

The transcription factor activator protein (AP)-2 $\alpha$ has been shown to regulate many of the genes that are involved in proliferation, cell cycle regulation, apoptosis, adhesion, including $\mathrm{CDH} 1$, and invasion. The loss of AP- $2 \alpha$ has been reported to be a common molecular abnormality in human high-grade astrocytomas (Heimberger et al., 2005). These data match perfectly with our results, where we observed lower levels of $\mathrm{CDH} 1$ expression in high-grade astrocytomas. Some have shown that re-expression of AP- $2 \alpha$ results in a 4-fold increase in the activity of E-cadherin gene transcription (Schwartz et al., 2007).

This mechanism, besides the aberrant methylation of the $C D H 1$ promoter, seems to be important in the decrease or loss of E-cadherin expression in neuroepithelial tumors. The decrease of E-cadherin expression seems to be associated with a transcriptional stimulation of another cadherin, $\mathrm{N}$-cadherin. Decrease of E-cadherin expression and $\mathrm{N}$-cadherin stimuli is first 
observed during gastrulation, associated with loss of epithelial structure and gain of migratory characteristics (Van Aken et al., 2001). Increase of expression of N-cadherin is commonly reported in neuroepithelial tumors that show a loss of E-cadherin expression (Asano et al., 2000; Utsuki et al., 2002, 2004). Brain tumors with high expression of N-cadherin have been found to have marked invasive proprieties and poor prognosis (Utsuki et al., 2004). These features may be a signature for undifferentiated tumors such as medulloblastoma and glioblastoma.

Kaplan-Meier and log-rank analysis showed no difference in the survival proportion of subjects with high E-cadherin expression compared to those with low E-cadherin expression. However, others have reported that subjects with low expression of $\mathrm{CDH} 1$ gene in epithelial tumors have a worse prognosis (Chen et al., 2003).

We found that E-cadherin is expressed differently among neuroepithelial tumors. Our finding suggests that a decrease in E-cadherin gene expression may be a hallmark of malignancy and it may be correlated with progression and dissemination of brain tumors. Moreover, further investigation is needed to clarify the mechanisms involved in the decrease/loss of $\mathrm{CDH1}$ expression in neuroepithelial tumors.

\section{ACKNOWLEDGMENTS}

We thank Dr. Renata Canalle for critical reading of this manuscript and useful suggestions. Research supported by the Brazilian governmental agencies FAPESP (04/12133-6), FAEPA and CNPq.

\section{REFERENCES}

Asano K, Kubo O, Tajika Y, Takakura K, et al. (2000). Expression of cadherin and CSF dissemination in malignant astrocytic tumors. Neurosurg. Rev. 23: 39-44.

Becker KF, Rosivatz E, Blechschmidt K, Kremmer E, et al. (2007). Analysis of the E-cadherin repressor Snail in primary human cancers. Cells Tissues Organs 185: 204-212.

Behin A, Hoang-Xuan K, Carpentier AF and Delattre JY (2003). Primary brain tumours in adults. Lancet 361: 323-331.

Cavallaro U and Christofori G (2001). Cell adhesion in tumor invasion and metastasis: loss of the glue is not enough. Biochim. Biophys. Acta 1552: 39-45.

Chen HC, Chu RY, Hsu PN, Hsu PI, et al. (2003). Loss of E-cadherin expression correlates with poor differentiation and invasion into adjacent organs in gastric adenocarcinomas. Cancer Lett. 201: 97-106.

Frühwald MC, O'Dorisio MS, Dai Z, Tanner SM, et al. (2001). Aberrant promoter methylation of previously unidentified target genes is a common abnormality in medulloblastomas - implications for tumor biology and potential clinical utility. Oncogene 20: 5033-5042.

Garinis GA, Menounos PG, Spanakis NE, Papadopoulos K, et al. (2002). Hypermethylation-associated transcriptional silencing of E-cadherin in primary sporadic colorectal carcinomas. J. Pathol. 198: 442-449.

Graziano F, Humar B and Guilford P (2003). The role of the E-cadherin gene (CDH1) in diffuse gastric cancer susceptibility: from the laboratory to clinical practice. Ann. Oncol. 14: 1705-1713.

Guarino M, Rubino B and Ballabio G (2007). The role of epithelial-mesenchymal transition in cancer pathology. Pathology 39: 305-318.

Hazan RB, Qiao R, Keren R, Badano I, et al. (2004). Cadherin switch in tumor progression. Ann. N. Y. Acad. Sci. 1014: 155-163.

Heimberger AB, McGary EC, Suki D, Ruiz M, et al. (2005). Loss of the AP-2alpha transcription factor is associated with the grade of human gliomas. Clin. Cancer Res. 11: 267-272.

Hirohashi S (1998). Inactivation of the E-cadherin-mediated cell adhesion system in human cancers. Am. J. Pathol. 153: 333-339.

Howng SL, Wu CH, Cheng TS, Sy WD, et al. (2002). Differential expression of Wnt genes, beta-catenin and E-cadherin in human brain tumors. Cancer Lett. 183: 95-101. 
Khatua S, Peterson KM, Brown KM, Lawlor C, et al. (2003). Overexpression of the EGFR/FKBP12/HIF-2alpha pathway identified in childhood astrocytomas by angiogenesis gene profiling. Cancer Res. 63: 1865-1870.

Lombaerts M, van Wezel T, Philippo K, Dierssen JW, et al. (2006). E-cadherin transcriptional downregulation by promoter methylation but not mutation is related to epithelial-to-mesenchymal transition in breast cancer cell lines. $\mathrm{Br}$. $J$. Cancer 94: 661-671.

Louis DN, Ohgaki H, Wiestler OD, Cavenee WK, et al. (2007). The 2007 WHO classification of tumours of the central nervous system. Acta Neuropathol. 114: 97-109.

Masterson J and O'Dea S (2007). Posttranslational truncation of E-cadherin and significance for tumour progression. Cells Tissues Organs 185: 175-179.

Mol AJ, Geldof AA, Meijer GA, van der Poel HG, et al. (2007). New experimental markers for early detection of high-risk prostate cancer: role of cell-cell adhesion and cell migration. J. Cancer Res. Clin. Oncol. 133: 687-695.

Munaut C, Noel A, Hougrand O, Foidart JM, et al. (2003). Vascular endothelial growth factor expression correlates with matrix metalloproteinases MT1-MMP, MMP-2 and MMP-9 in human glioblastomas. Int. J. Cancer 106: 848-855.

Perego C, Vanoni C, Massari S, Raimondi A, et al. (2002). Invasive behaviour of glioblastoma cell lines is associated with altered organisation of the cadherin-catenin adhesion system. J. Cell Sci. 115: 3331-3340.

Pingoud-Meier C, Lang D, Janss AJ, Rorke LB, et al. (2003). Loss of caspase-8 protein expression correlates with unfavorable survival outcome in childhood medulloblastoma. Clin. Cancer Res. 9: 6401-6409.

Pokutta S and Weis WI (2007). Structure and mechanism of cadherins and catenins in cell-cell contacts. Annu. Rev. Cell Dev. Biol. 23: 237-261.

Pötter R, Czech TH and Dieckmann I (1998). Tumors of the central nervous system. In: Cancer in Children: Clinical Management (Voute PA, Kalifa C and Barrett A, eds.). Oxford University Press, New York, 175-180.

Redies C (2000). Cadherins in the central nervous system. Prog. Neurobiol. 61: 611-648.

Russo C, Pellarin M, Tingby O, Bollen AW, et al. (1999). Comparative genomic hybridization in patients with supratentorial and infratentorial primitive neuroectodermal tumors. Cancer 86: 331-339.

Schwartz B, Melnikova VO, Tellez C, Mourad-Zeidan A, et al. (2007). Loss of AP-2alpha results in deregulation of E-cadherin and MMP-9 and an increase in tumorigenicity of colon cancer cells in vivo. Oncogene 26: 4049-4058.

Schwechheimer K, Zhou L and Birchmeier W (1998). E-cadherin in human brain tumours: loss of immunoreactivity in malignant meningiomas. Virchows Arch. 432: 163-167.

Scrideli CA, Cazzaniga G, Fazio G, Pirola L, et al. (2003). Gene expression profile unravels significant differences between childhood and adult $\mathrm{Ph}+$ acute lymphoblastic leukemia. Leukemia 17: 2234-2237.

Shapiro L, Love J and Colman DR (2007). Adhesion molecules in the nervous system: structural insights into function and diversity. Annu. Rev. Neurosci. 30: 451-474.

Strother DR, Pollack IF, Gisher PG, Hunter JV, et al. (2002). Tumors of the central nervous system. In: Principles and Practice of Pediatric Oncology (Pizzo PA and Poplack DG, eds.). Lippincott Williams and Wilkins, Philadelphia, 751-824.

Takeichi M (1991). Cadherin cell adhesion receptors as a morphogenetic regulator. Science 251: 1451-1455.

Uhlmann K, Rohde K, Zeller C, Szymas J, et al. (2003). Distinct methylation profiles of glioma subtypes. Int. J. Cancer 106: 52-59.

Utsuki S, Sato Y, Oka H, Tsuchiya B, et al. (2002). Relationship between the expression of E-, N-cadherins and betacatenin and tumor grade in astrocytomas. J. Neurooncol. 57: 187-192.

Utsuki S, Oka H, Sato Y, Tsutiya B, et al. (2004). E, N-cadherins and beta-catenin expression in medulloblastoma and atypical teratoid/rhabdoid tumor. Neurol. Med. Chir. 44: 402-406.

Van Aken E, De Wever O, Correia da Rocha AS and Mareel M (2001). Defective E-cadherin/catenin complexes in human cancer. Virchows Arch. 439: 725-751.

Wijnhoven BP, Dinjens WN and Pignatelli M (2000). E-cadherin-catenin cell-cell adhesion complex and human cancer. Br. J. Surg. 87: 992-1005.

Yagi T and Takeichi M (2000). Cadherin superfamily genes: functions, genomic organization, and neurologic diversity. Genes Dev. 14: 1169-1180.

Yi Kim D, Kyoon JJ, Kyu PY, Yeob RS, et al. (2007). E-cadherin expression in early gastric carcinoma and correlation with lymph node metastasis. J. Surg. Oncol. 96: 429-435.

Yu J, Zhang H, Gu J, Lin S, et al. (2004). Methylation profiles of thirty four promoter-CpG islands and concordant methylation behaviours of sixteen genes that may contribute to carcinogenesis of astrocytoma. BMC Cancer 4: 65 . 